\title{
Activation of extracellular signal-regulated protein kinase 5 downregulates FasL upon osmotic stress
}

\author{
X Wang ${ }^{1,4}, K_{\text {K Finegan }}{ }^{1,4}$, AC Robinson ${ }^{1}$, L Knowles ${ }^{1}$, \\ R Khosravi-Far ${ }^{2}$, KA Hinchliffe ${ }^{1}$, RP Boot-Handford ${ }^{3}$ \\ and $C$ Tournier ${ }^{*, 1}$ \\ ${ }^{1}$ Faculty of Life Sciences, University of Manchester, Manchester, UK \\ 2 Department of Pathology, Harvard Medical School, Boston MA, USA \\ ${ }^{3}$ Wellcome Trust Center for Cell Matrix, University of Manchester, \\ Manchester, UK \\ ${ }^{4}$ The first two authors contributed equally to this study \\ * Corresponding author: C Tournier, Faculty of Life Sciences, University of \\ Manchester, Michael Smith Building, Oxford Road, Manchester M13 9PT, UK. \\ Tel: +44161275 5417; Fax: + 44161275 5082; \\ E-mail: cathy.tournier@manchester.ac.uk
}

Received 24.11.05; revised 16.3.06; accepted 13.4.06; published online 19.5.06 Edited by S Nagata

\begin{abstract}
Extracellular signal-regulated protein kinase (ERK) 5 is a mitogen-activated protein kinase (MAPK) that is activated by dual phosphorylation via a unique MAPK/ERK kinase 5, MEK5. The physiological importance of this signaling cascade is underscored by the early embryonic death caused by the targeted deletion of the erk5 or the mek5 genes in mice. Here, we have found that ERK5 is required for mediating the survival of fibroblasts under basal conditions and in response to sorbitol treatment. Increased Fas ligand (FasL) expression acts as a positive feedback loop to enhance apoptosis of ERK5- or MEK5deficient cells under conditions of osmotic stress. Compared to wild-type cells, erk5-/ - and mek5-/ - fibroblasts treated with sorbitol display a reduced protein kinase B (PKB) activity associated with increased Forkhead box 03a (Foxo3a) activity. Based on these results, we conclude that the ERK5 signaling pathway promotes cell survival by downregulating FasL expression via a mechanism that implicates PKB-dependent inhibition of Foxo3a downstream of phosphoinositide 3 kinase. Cell Death and Differentiation (2006) 13, 2099-2108. doi:10.1038/sj.cdd.4401969; published online 19 May 2006
\end{abstract}

Keywords: ERK5; FasL; MEK5; PKB; Bid; Bim

Abbreviations: ERK, extracellular signal-regulated protein kinase; FasL, Fas ligand; Foxo3a, Forkhead box O3a; MAPK, mitogen-activated protein kinase; MEF2, myocyte enhancer factor 2; MEFs, mouse embryonic fibroblasts; MEK, MAPK/ERK kinase; PDK1, 3-phosphoinositide-dependent protein kinase-1; $\mathrm{PI} 3 \mathrm{~K}$, phosphoinositide 3 kinase; PKB, protein kinase B; mTor, mammalian target of rapamycin protein kinase

\section{Introduction}

The mitogen-activated protein kinases (MAPK) constitute a family of serine/threonine protein kinases involved in the regulation of many cellular functions, including proliferation, survival, and apoptosis. ${ }^{1}$ There are at least four subfamilies positioned at the end of distinct signaling cascades that include a MAPK kinase and a MAPK kinase kinase. ${ }^{1}$ Although these pathways transmit signals independently of each other, cross talk occurs between the different modules thereby leading to a greater level of complexity that allows the fine-tuning of responses to extracellular stimuli. MAPK modules have been associated with different biological responses. For example, the extracellular signal-regulated protein kinase (ERK) subfamilies 1,2 , and 5 are mostly associated with cell proliferation and survival, wereas c-Jun $\mathrm{N}$-terminus protein kinase (JNK) and p38 MAPK are mainly activated in response to cytokines and extracellular stresses and mediate apoptosis.

ERK5 is more than twice the size of the other MAPKs owing to a very large C-terminal domain. ${ }^{2,3}$ Its activity is stimulated in response to growth factors and hyperosmolarity via the MAPK/ERK kinase, MEK5. ${ }^{4}$ Little is known about the downstream targets of ERK5. The best-characterized substrates are the transcription factors of the myocyte enhancer factor (MEF) family. ${ }^{5,6}$ Phosphorylation of MEF2C by ERK5 enhances its transcriptional activity and subsequently increases expression of the AP-1 family member, c-Jun. ${ }^{5}$ Consistent with this study, we have recently demonstrated that ERK5 is selectively required for the regulation of c-Jun expression following EGF stimulation of the cells but not by UV radiation. ${ }^{7}$

Clues as to the physiological role of the ERK5 signaling pathway have recently been provided by the targeted deletion of the erk5 and mek5 genes in mice. ${ }^{4,8}$ The analysis of mutant mice in which the erk5 gene can be conditionally deleted revealed that the requirement of ERK5 for the survival of endothelial cells is responsible for the cardiovascular defect observed in erk5-/- and mek5-/- embryos. ${ }^{9}$ The ERK5 signaling pathway protects endothelial cells from apoptosis by phosphorylating and inhibiting the $\mathrm{Bcl}-2$ family member Bad. ${ }^{10}$ Evidence that ERK5 contributes to mediating neuronal survival in response to growth factors has also been reported. ${ }^{11}$

The Ser/Thr protein kinase B (PKB, also known as Akt) is another key mediator of signal transduction implicated in protecting cells against death. Activation of PKB is mediated by dual phosphorylation at Thr308 and Ser473. ${ }^{12}$ Phosphorylation at Thr308 is mediated by the 3-phosphoinositide (PtIns)-dependent protein kinase-1 (PDK1) via a PtIns 3 kinase (PI3K)-dependent mechanism, ${ }^{13}$ whereas Ser473 is a target for the mammalian target of rapamycin protein kinase, mTor. ${ }^{14}$ The survival function of PKB can be explained by its ability to inhibit the activity of proapoptotic proteins including $\mathrm{Bad}^{15}$ and the Forkhead transcription factor box O3a (Foxo3a formerly called FKHRL1). ${ }^{16}$ In both cases, phosphorylation provides a mechanism to sequester the proteins in the cytoplasm thereby blocking their apoptotic effect. 
Here we have investigated how the ERK5 signaling pathway regulates the survival response of fibroblasts to osmotic stress. Our data indicate that the ERK5 cascade suppresses FasL expression by inhibiting its transcription via Foxo3a. Further experiments demonstrate that PKB activation following sorbitol stimulation is impaired in the absence of ERK5 or MEK5. Overall, this study establishes for the first time a functional cross talk between the ERK5 and the PKB signaling pathways to prevent enhancement of cell death via increased FasL expression.

\section{Results}

\section{Deletion of the erk5 gene causes apoptosis}

The role of ERK5 in cell survival was examined by testing the effect of erk5 gene deletion in homozygous erk5loxP mouse embryonic fibroblasts (MEFs) infected with an adenovirus encoding Cre (AdCre). The strategy employed to create the erk5loxP mutant mice is provided as Supplementary information (Figure S1). Immunoblot analysis of the cell lysates $72 \mathrm{~h}$ postinfection demonstrated the gradual loss of the ERK5 protein following increased Cre expression (Figure 1a). Future experiments were performed with adenoviruses at
100 multiplicity of infection (MOI) to induce the complete deletion of the erk5 gene. A time course analysis of infection showed that caspase 3 activity was significantly increased in erk5loxP/loxP but not in wild-type MEFs infected with AdCre (Figure 1b). The slight effect of AdCre in the erk5+/loxP MEFs indicates a low level of cell death associated with heterozygous deletion of the erk5 gene. Hereinafter, the erk5loxP/lox $P$ fibroblasts were immortalized and referred to as erk5+/+ or erk5-/- MEFs depending on whether they were infected with a control virus encoding green fluorescence protein (GFP) or Cre, respectively.

Immunoblot analysis confirmed the requirement of ERK5 to suppress the activation of caspase 3 under basal conditions, and also in response to sorbitol, a potent inducer of apoptosis and a strong activator of ERK5 (Figure 1c). A reduction of the inactive p32 proenzyme together with the appearance of the active $\mathrm{p} 17$ product was detected $3 \mathrm{~h}$ earlier in erk5loxP/lox $P$ MEFs infected with Cre (-/-) than in erk5loxP/loxP MEFs infected with GFP $(+/+)$. More strikingly, sorbitol induced the proteolytic cleavage of the proapoptotic Bcl-2 family member Bid to generate the death-promoting fragment tBid, only in absence of ERK5 (Figure 1c). The level of tBid was maximal after $1 \mathrm{~h}$ stimulation consistent with a contribution of Bid upstream of caspase 3.
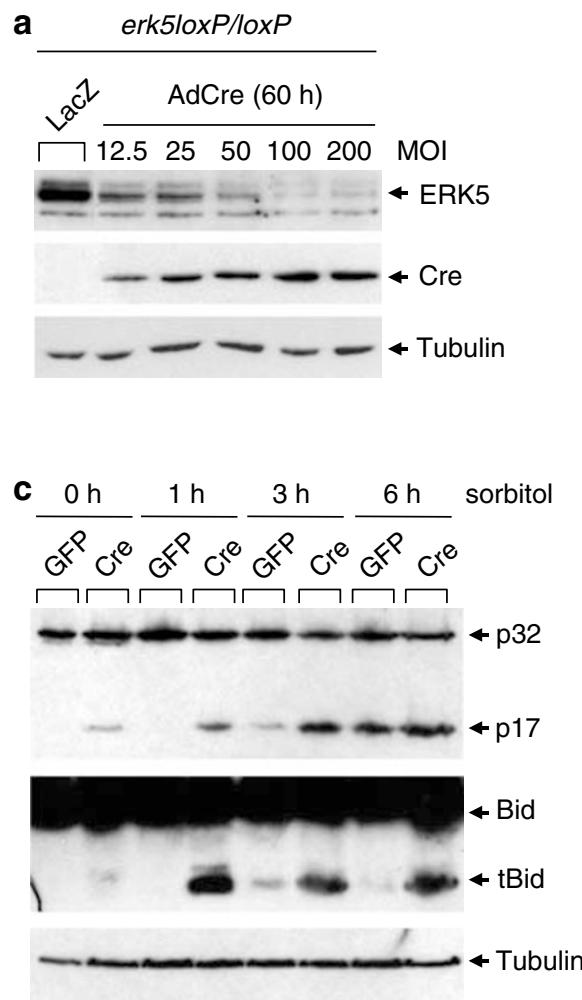
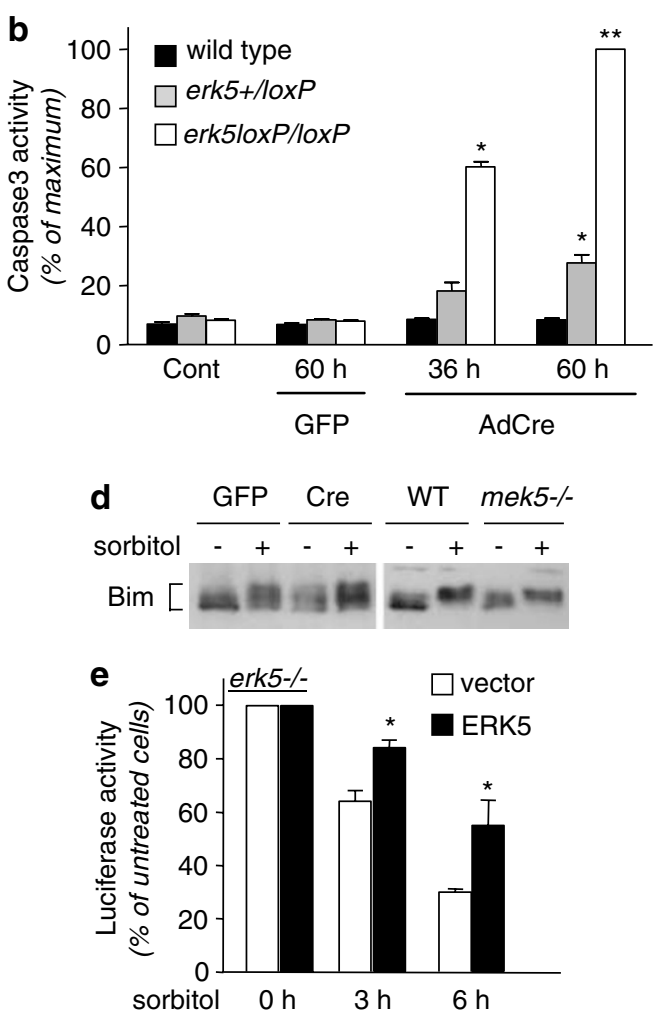

Figure 1 erk5 gene deletion sensitizes fibroblasts to apoptosis. MEFs were not infected (Cont), or infected with a control virus (lacZ or GFP) or with AdCre befor being incubated with sorbitol where indicated. (a, c, d) Extracts $(50 \mu \mathrm{g})$ were analyzed for ERK5, Cre, caspase 3, Bid, and Bim expression by immunoblot analysis using specific antibodies. The detection of tubulin expression was performed to monitor protein loading. Similar results were obtained in two to three independent experiments. (b) Caspase 3 activity was measured by caspase assay. The data correspond to the mean \pm S.E. of three independent experiments performed in duplicate. $P$-values relative to wild-type sample are indicated by asterisks: ${ }^{\star} P<0.05$; ${ }^{* \star} P \leqslant 0.001$. (e) erk5-/- fibroblasts were cotransfected with an empty vector (vector) or a vector encoding wild-type ERK5, and luciferase (pCMV-luc) to monitor cell viability. At $36 \mathrm{~h}$ after transfection, the cells were treated with sorbitol for the indicated times before being lysed and the luciferase activity was measured. The values are normalized to the protein content. The data expressed as \% of treated versus untreated cells correspond to the mean \pm S.E. of three independent experiments. ${ }^{*} P<0.0001$ indicates significant increase in survival of cells transfected with ERK5 
Bim is another BH3-only protein of the Bcl-2 family implicated in the intrinsic cell death signaling pathway. It becomes hyperphosphorylated upon apoptotic stress and dissociates from the microtubule-associated dynein motor complex where it is normally sequestered. ${ }^{17}$ Deletion of the erk5 gene under basal conditions or incubation of the fibroblasts with sorbitol caused distinct electrophoretic mobility shifts indicative of Bim being phosphorylated (Figure 1d). Unlike Bid cleavage, no significant difference was observed in the ability of sorbitol to phosphorylate Bim in wild-type and erk5-/- or mek5-/- MEFs.

Cell viability assay that employs a luciferase plasmid showed that ectopic expression of ERK5 was able to protect fibroblasts against sorbitol toxicity (Figure 1e). erk5-/- MEFs transfected with a vector encoding ERK5 displayed 15 and $45 \%$ decrease in luciferase activity, as compared to 35 and $70 \%$ when the cells were transfected with an empty vector, following 3 and $6 \mathrm{~h}$ sorbitol treatment, respectively. This agrees with our previous data showing that mek5-/- MEFs were more sensitive than the wild-type cells to the toxic effect of sorbitol. ${ }^{4}$ Similar to the phenotypic defect observed in the erk5-/- fibroblasts, enhanced cell death displayed by MEFs lacking MEK5 correlated with increased caspase 3 and Bid cleavage (Supplementary Figure S2).

Together, these studies suggest that activation of the proapoptotic activity of Bim and Bid may be responsible for the abnormal cell death phenotype displayed by the erk5-/MEFs under basal conditions and in response to stress, respectively.

\section{ERK5 protects cells by inhibiting FasL expression}

Cleavage of Bid plays a major role in mediating the apoptotic response of cells to factors acting via death receptors, including FasL. As FasL has previously been shown to contribute to stress-induced apoptosis, we tested the hypothesis that an increase in FasL expression was responsible for sorbitol-induced Bid cleavage. Real-time PCR analysis showed that sorbitol enhanced the levels of fas $L$ mRNA in the erk5-/- and mek5-/- MEFs compared to the wild-type $(+/+)$ cells (Figure $2 a$ and $b)$. Consistently, around $60 \%$ FasL-positive ERK5- or MEK5-deficient MEFs were detected upon sorbitol stimulation compared to $13-20 \%$ positive cells of the corresponding wild-type genotype (Figure $2 \mathrm{c}$ and d).

The importance of FasL to mediate the apoptotic effect of sorbitol was demonstrated by the ability of a neutralizing anti-FasL antibody to reduce the activation of caspase 3 (Figure $2 \mathrm{e}$ and $\mathrm{f}$ ). The greatest inhibition (around 75\%) was detected in the mek5-/- MEFs where caspase 3 activation was decreased to a similar level as in the wild-type cells (Figure 2f). The lower level of inhibition (around 45\%) observed in the erk5-/- MEFs is explained by the inability of the antibody to block Fas-independent caspase 3 activation caused by erk5 gene deletion (Figure 2e). Consistently, absence of ERK5 had no effect on FasL expression under basal conditions (time 0) (Figure 2a and c).

Together, these experiments indicate that FasL produced in response to sorbitol acts in a positive feedback loop to enhance the death of ERK5- or MEK5-deficient cells under conditions of osmotic stress.

\section{ERK5 is required to inhibit Foxo3 activity}

A number of response elements have been identified in the promoter of the fas $L$ gene including binding sites for the transcription factors C-Jun and Foxo3a. ${ }^{16,18,19}$ We found that c-Jun mRNA and protein expression was downregulated following sorbitol treatment of the cells (unpublished data). Furthermore, we could not detect any electrophoretic mobility shift typical of the protein being phosphorylated. We concluded that c-Jun was unlikely to be involved in the transcriptional regulation of the fas $L$ gene under osmotic stress conditions. This prompted us to test the hypothesis that the requirement of the ERK5 signaling pathway to inhibit Foxo3a activity was responsible for the upregulation of FasL expression in cells deficient in ERK5 or MEK5 expression. Real-time PCR analysis showed similar levels of foxo3a mRNA in wild-type, erk5-/-, and mek5-/- MEFs (Figure 3a). Immunoblot analysis demonstrated that the deletion of the erk5 or the mek5 genes decreases both the basal level and sorbitol-induced phosphorylation of Foxo3a at Thr32 (Figure 3b). The phosphorylation of Foxo3a at Thr32 leads to its association with 14-3-3, and thus, its sequestration in the cytoplasm. ${ }^{16}$

The ability of the ERK5 signaling pathway to control Foxo3a activity was examined by luciferase reporter assay (Figure 3c and d). Wild-type fibroblasts were cotransfected with a luciferase reporter plasmid containing three Foxo3a-binding sites from the FasL promoter (FHRE-Luc, 16), together with an empty plasmid (vector) or a plasmid encoding wildtype (WT) or triple mutant (TM) Foxo3a in which Thr32, Ser253, and Ser315 were converted to Ala residues. ${ }^{16}$ Foxo3a TM is constitutively expressed in the nucleus and acts as a dominant active mutant. A pRL-Tk plasmid encoding Renilla luciferase was employed for monitoring transfection efficiency. The results showed that sorbitol increased FHRE-Luc activity in cells overexpressing Foxo3a WT to a similar level as overexpressed Foxo3a TM (Figure 3c). Unlike in wild-type cells, sorbitol was capable of stimulating endogenous Foxo3a activity in ERK5- or MEK5-deficient fibroblasts transfected with FHRE-Luc (Figure 3d). The wildtype phenotype was restored following the reintroduction of ERK5 or MEK5 in erk5-/- or mek5-/- fibroblasts, respectively (Figure $3 d$ ). The requirement of the ERK5 signaling pathway to suppress Foxo3a-dependent transcription of the fasl gene was further demonstrated by chromatin immunoprecipitation (ChIP) assay (Figure 3e). The experiment showed that sorbitol induced the binding of Foxo3a to the promoter of the fas $L$ gene in the erk5-/- or mek5-/- MEFs $3 \mathrm{~h}$ earlier than in the wild-type cells.

Together, these studies suggest that the ERK5 signaling pathway blocked sorbitol-induced transcription via Foxo3a by preventing the nuclear translocation of Foxo3a.

\section{Cross talk between the ERK5 and the PKB signaling pathways}

Evidence suggested that recombinant Foxo3a was not a substrate of ERK5 in vitro (unpublished data). As PKBdependent phosphorylation of Foxo3a at Thr32 inhibits FasL expression, ${ }^{16}$ we examined the possibility that ERK5 was 

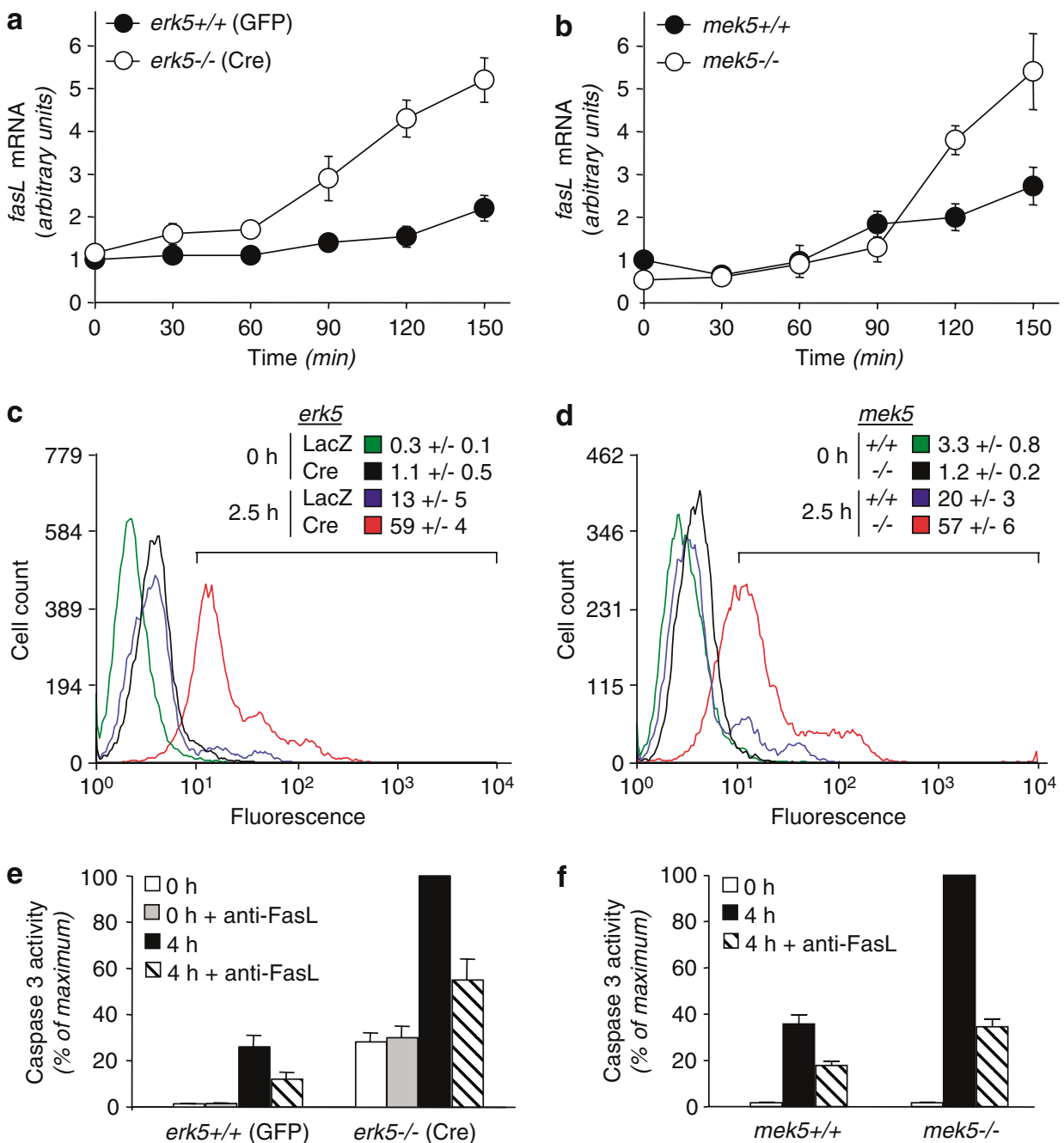

Figure 2 Enhanced sensitivity of ERK5- or MEK5-deficient fibroblasts to sorbitol is caused by increased FasL expression. MEFs were stimulated with sorbitol for the indicated times. $(\mathbf{a}, \mathbf{b})$ Total RNA was extracted and the amounts of fas $L$ transcript were measured by quantitative PCR. The data correspond to the mean $\pm S$.E. of three independent experiments performed in duplicate. (c, d) FasL expression was assessed by flow cytometry. The percent of Fas $\mathrm{p}$ positive cells $\pm S$.E. of three independent experiments is indicated. (e, f) Where indicated, the cells were incubated in the presence of a neutralizing anti-FasL antibody. Caspase 3 activity was measured by caspase assay. The data correspond to the mean \pm S.D. of duplicate samples. Similar results were obtained in two independent experiments

required for sorbitol-induced PKB activation. The results showed that the deletion of the erk5 or the mek5 genes prevented sorbitol from activating PKB (Figure $4 a$ and b). Immunoblot analysis using phospho-specific antibodies demonstrated that absence of MEK5 or ERK5 affected the ability of sorbitol to increase the phosphorylation of PKB at Thr308 and Ser473 (Figure 4c). Consistently, overexpression of a dominant active mutant of MEK5 (MEK5DA) in wild-type fibroblasts expressing Ha-tagged PKB caused a 2.5-fold increased in PKB activity compared to five-fold following sorbitol treatment (Figure 4d). The requirement of the ERK5 signaling pathway in regulating PKB activity was specific in response to stress as we found no difference in the ability of IGF1 to activate PKB in wild-type and mek5-/- MEFs (Supplementary Figure S3).

PDK1 is the protein kinase that phosphorylates PKB at Thr308 via a PI3K-dependent mechanism. ${ }^{13}$ We found that MEK5 or sorbitol-induced PKB activation was blocked by the pretreatment of the cells with wortmannin or LY294002, two well-characterized PI3K inhibitors (Figures $4 d$ and $5 a$ ). The requirement of $\mathrm{PI} 3 \mathrm{~K}$ in regulating the apoptotic response of MEFs under conditions of osmotic stress was demonstrated by the ability of wortmannin to increase the levels of fasL mRNA in wild-type cells treated with sorbitol (Figure $5 b$ ). Consistently, wortmannin enhanced the number of FasLpositive cells following sorbitol treatment (Figure 5c). Sorbitolincreased PI3K activity was not affected by the deletion of the erk5 of mek5 genes (Figure $5 \mathrm{~d}-\mathrm{g}$ ), indicating that the ERK5 signaling pathway promotes cell survival by downregulating FasL expression via a mechanism downstream of PI3K.

\section{Discussion}

This study is the first that investigates the molecular mechanism by which the ERK5 signaling pathway promotes 
a

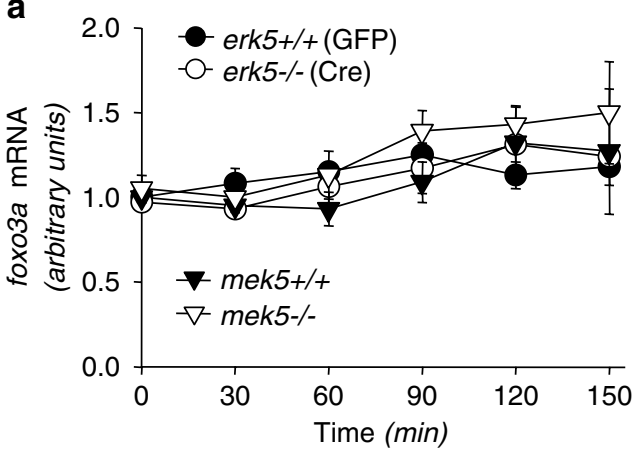

C

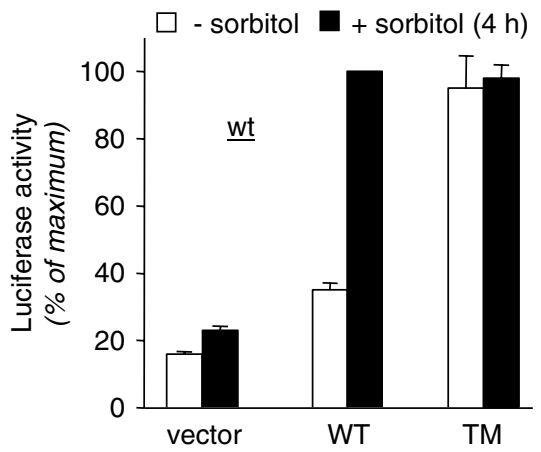

e

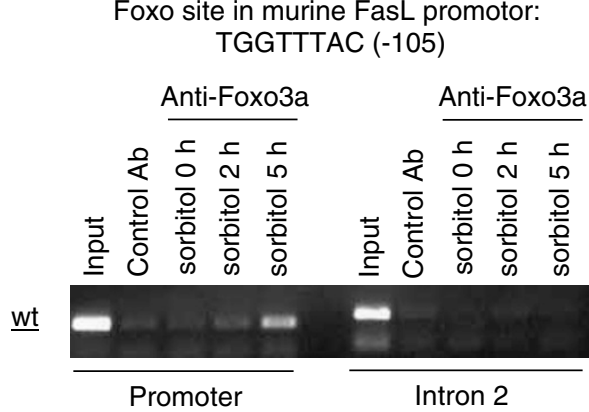

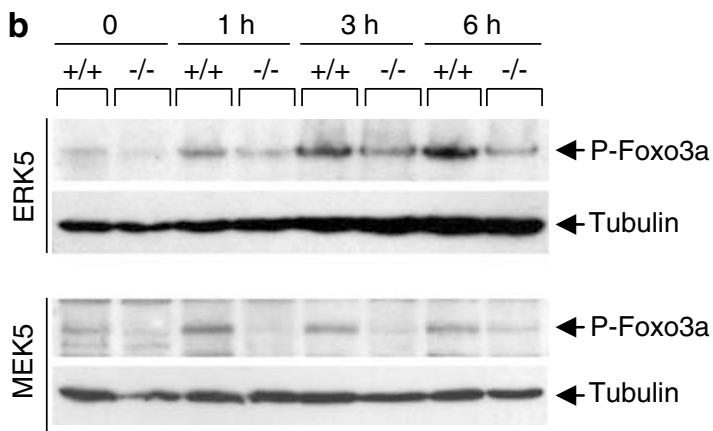

d $\quad \square$-sorbitol $\square$ + sorbitol (2h)
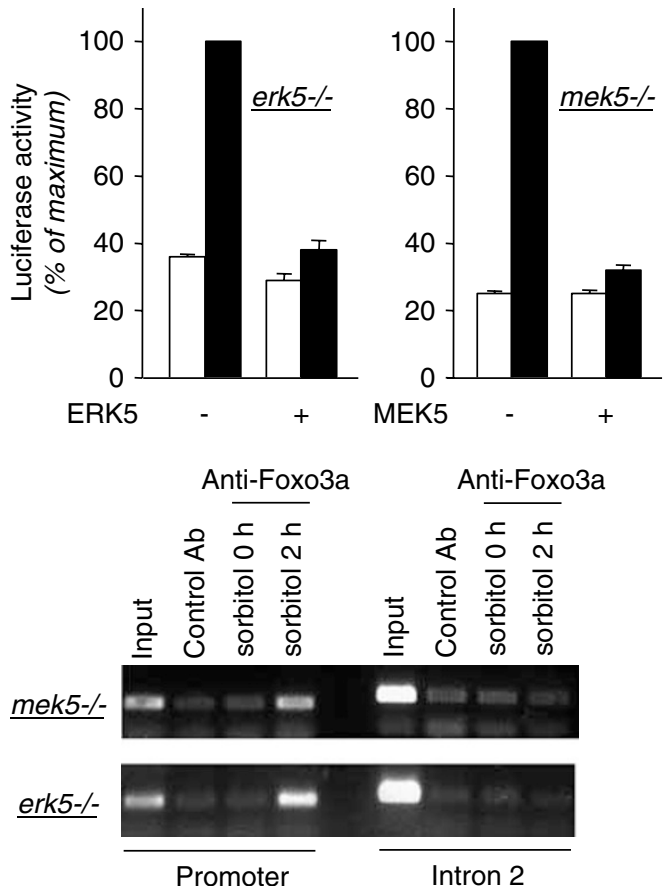

Figure 3 The ERK5 pathway is required to inhibit sorbitol-induced transcription via Fox03a. MEFs were treated with sorbitol for the indicated times. (a) Total RNA was extracted and the amounts of foxo3a transcript were measured by quantitative PCR. The data correspond to the mean \pm S.E. of three independent experiments performed in duplicate. (b) Extracts $(50 \mu \mathrm{g})$ were analyzed for phosphorylation of Foxo3a at Thr32 by immunoblot analysis using a specific antibody (P-Foxo3a). The detection of tubulin expression was performed to monitor protein loading. Similar results were obtained in two independent experiments. (c) Wild-type fibroblasts were transiently cotransfected with the reporter plasmid FHRE-luc together with an empty vector (vector) or a vector encoding wild-type (WT) or dominant active mutant (TM) Fox03a. (d) erk5-/ - and mek5-/- fibroblasts were transiently cotransfected with the reporter plasmid FHRE-luc with $(+)$ or without $(-)$ an expression vector encoding ERK5 or MEK5. Foxo3a-dependent transcriptional activity was measured by the Dual-Luciferase reporter assay system (c, d). The data correspond to the mean \pm S.E. of three independent experiments performed in duplicate. (e) Chromatin immunoprecipitation was performed using a Foxo3a-specific or IgG control antibody, followed by PCR-based amplification with primers corresponding to the promoter region $(-283 /-63)$ and intron $2(+2,439 /+2,720)$ of the mouse fas/ gene. The sequence of the putative Foxo-binding site present in the promoter region at position -105 is indicated. Similar results were obtained in two independent experiments

the survival of mitotic cells (Figure 6). We found that, under basal conditions, the loss of ERK5 expression correlates with increased death of MEFs. This contradicts previous studies that reported no noxious effect associated with erk5 gene deletion in fibroblasts. ${ }^{7,9,20}$ The explanation for such discrepancy may lie in the origin of the cells. Hayashi et al. ${ }^{9}$ extracted their fibroblasts from adult mice, whereas we have used embryonic cells. The other two studies have used ERK5-null MEFs that, unlike conditionally mutated cells that display a functional erk5 gene, may have been selected for compensatory survival mechanisms during the immortaliza- tion process. ${ }^{7,20}$ Similar modifications are likely to be found in the immortalized mek5-/- MEFs that did not exhibit any sign of cell death under basal conditions although displaying a low level of Foxo3a phosphorylation (Figure 3b). Alternatively, ERK5 expression may be more important than ERK5 activation in keeping alive proliferating cells. This is consistent with the demonstration that the intrinsic basal ERK5 activity is essential and sufficient to mediate survival of $\mathrm{Bcr} /$ Abl-expressing leukemia cells. ${ }^{21}$

In line with this idea, we found that the ERK5 cascade promotes cell survival under basal conditions and in response 

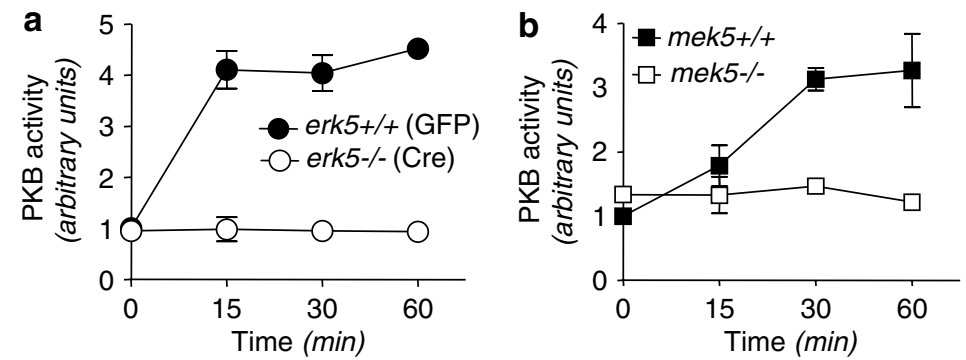

C
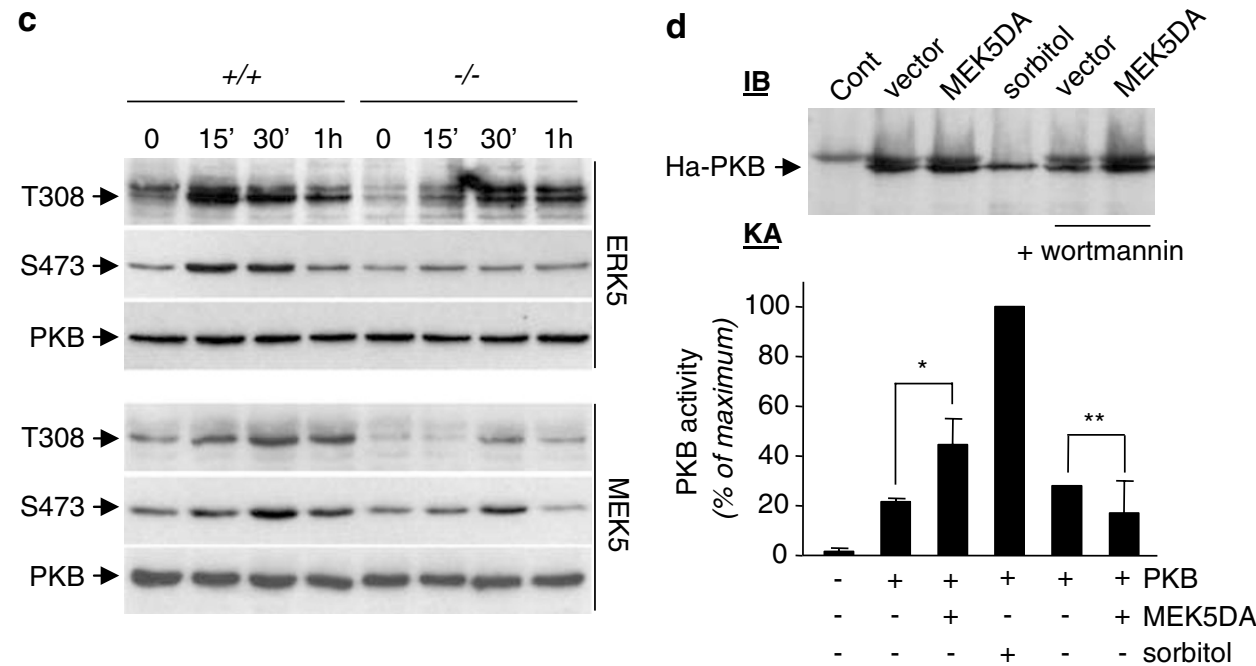

Figure 4 Regulation of PKB activity by the ERK5 signaling pathway. MEFs were incubated with sorbitol for the indicated times. (a, b) The activity of PKB was measured by protein kinase assay. The data correspond to the mean \pm S.E. of three independent experiments. (c) Extracts $(50 \mu \mathrm{g})$ of wild-type $(+/+)$ and homozygous ERK5- or MEK5-null (-/-) fibroblasts were analyzed for PKB expression and phosphorylation at Thr308 and Ser473 by immunoblot analysis using specific antibodies. Similar results were obtained in two to three independent experiments. (d) Wild-type fibroblasts were cotransfected with an expression vector encoding Ha epitope-tagged PKB with an empty vector $(-)$ or a vector encoding dominant active MEK5 $(+)$. Where indicated, the cells were stimulated with sorbitol for 30 min or pretreated with wortmannin. The expression of PKB in cell lysates was examined by immunoblot analysis (IB) using an anti-Ha antibody. PKB activity was measured by protein kinase assays (KA). Lysate of untransfected cells was used as control (Cont). The data correspond to the mean \pm SE of two independent experiments. $P$-values relative to basal PKB activity are indicated by asterisks: ${ }^{\star} P<0.01 ;{ }^{* \star} P>0.5$

to stress via different mechanisms. Increased FasL expression via upregulation of Foxo3a activity is responsible for enhanced cell death of the mek5-/- and erk5-/- MEFs treated with sorbitol. The regulation of $\mathrm{PKB}$ by the ERK5 pathway constitutes a likely mechanism by which ERK5 inhibits Foxo3a activity (Figure 4). This is consistent with the ability of wortmannin (Figure $5 b$ and $c$ ) and of $a$ dominant negative mutant of $\mathrm{PKB}^{22}$ to upregulate $\mathrm{FasL}$ in cells incubated with sorbitol. In contrast, the low basal Foxo3a phosphorylation associated with the loss of ERK5 did not correlate with increased fas $L$ transcript (Figure 2a). Consistently, the anti-FasL antibody was unable to reduce activation of caspase 3 caused by erk5 gene deletion (Figure 2e). Downstream targets of Foxo3a that may contribute to increasing cell death following the loss of ERK5 include Bim. ${ }^{23}$ However, no difference in Bim expression was observed between erk5-/-, mek5-/-, and wild-type cells treated or not with sorbitol (Figure 1e). The contribution of Foxo3a-induced Bim expression may be more relevant to the apoptotic response of hematopoietic cells and neurons deprived of trophic support. ${ }^{19,23}$ In contrast, the deletion of the erk5 gene in fibroblasts correlated with increased phosphorylation of Bim (Figure 1e). The requirement of ERK5 to inhibit Bim phosphorylation, thereby suppressing its proapoptotic activity, ${ }^{17}$ provides a possible mechanism by which ERK5 promotes cell survival under basal conditions.

The activation of PKB has been shown to modulate the death response of cells to a number of apoptotic signals. ${ }^{24}$ However, its function as a survival factor under osmotic stress conditions remains controversial as $\mathrm{PKB}$ has both been shown to be activated ${ }^{25}$ and inactivated ${ }^{26}$ by sorbitol treatment. Although cell-type differences may explain such discrepancy, the ability of sorbitol to increase PKB activity in fibroblasts has not been consistently observed. ${ }^{25,27}$ This could be explained by the fact that sorbitol is a weak activator of PKB making this effect difficult to detect. Our results clearly show that PKB activity is increased by sorbitol in fibroblasts (Figure 4). Overall, our data support the idea that PKB activation is functionally important to act as a brake on the apoptotic process. The specific requirement of the ERK5 signaling pathway in regulating PKB activity in response to sorbitol but not IGF1 (Supplementary Figure S3) underscores the existence of distinct mechanisms implicated in mediating survival signals in response to stress stimuli and growth factors.

Although MEK5DA-induced PKB activation was blocked by the pretreatment of the cells with wortmannin (Figure 4D), we 
a
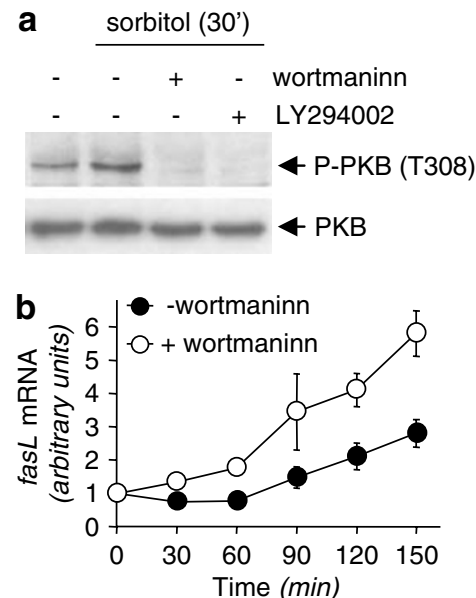

d

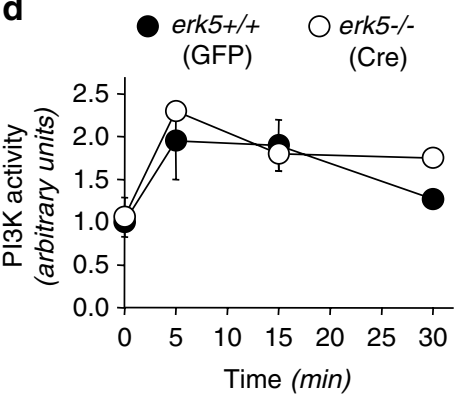

f

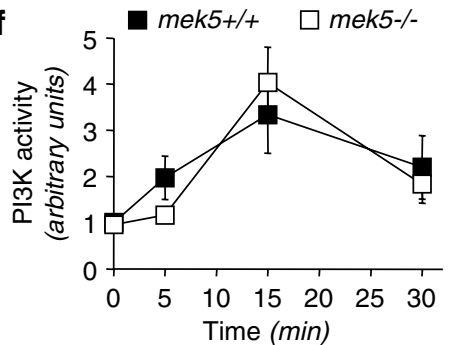

C
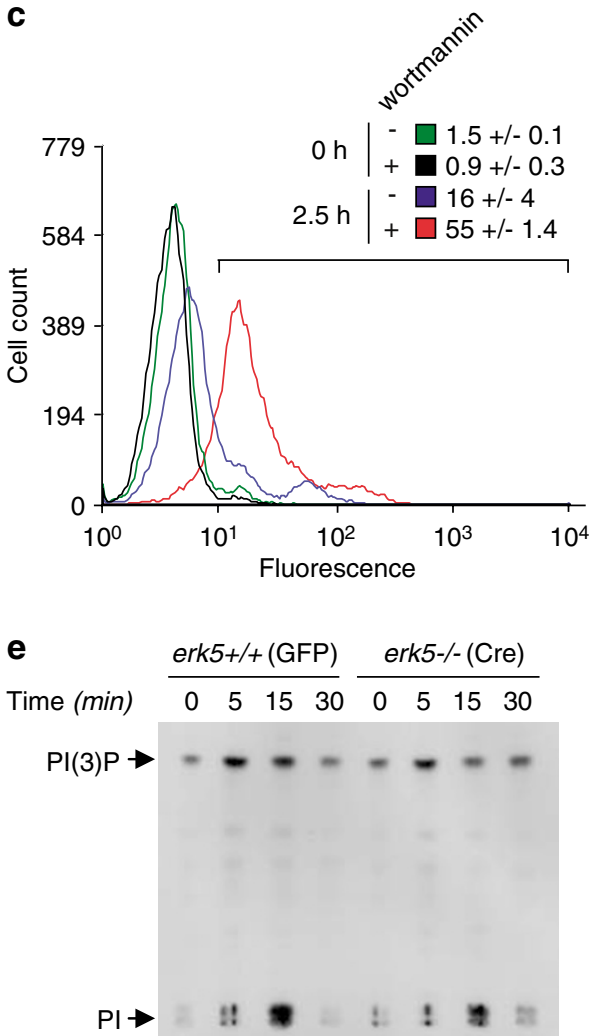

g

Time (min) $\quad 0 \quad 5 \quad 15 \quad 30 \quad 0 \quad 5 \quad 15 \quad 30$

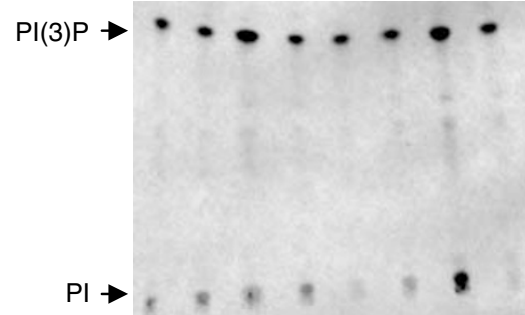

Figure 5 The ERK5 pathway does not affect sorbitol-induced PI3K activation. (a, b, c) Wild-type MEFs were pretreated (+) or not (-) with wortmannin or LY294002 before being incubated with sorbitol for the indicated times. Extracts $(50 \mu \mathrm{g})$ were analyzed for PKB expression and phosphorylation at Thr308 by immunoblot analysis using specific antibodies (a). The figure is representative of two independent experiments. Total RNA was extracted and the amounts of fasL transcript were measured by quantitative PCR (b). The data correspond to the mean \pm SE of three independent experiments performed in duplicate. FasL expression was assessed by flow cytometry (c). The percent of FasL-positive cells \pm S.E. of three independent experiments is indicated. $(\mathbf{d}, \mathbf{e}, \mathbf{f}, \mathbf{g}) \mathrm{MEFs}$ were incubated with sorbitol for the indicated times. The activity of PI3K was measured by kinase assay. The radioactivity incorporated into PI(3)P was quantitated. The data correspond to the mean $\pm \mathrm{S}$.E. of two to three independent experiments

found that sorbitol activated PI3K activity with no marked difference between wild-type and erk5-/_ or mek5-/- MEFs (Figure $5 d-g$ ). These data indicate that whereas active PI3K is required for optimal $\mathrm{PKB}$ activation, MEK5/ERK5 stimulate PKB activity by a mechanism downstream of PI3K. We have examined the ability of sorbitol to activate PDK1. The high basal level of PDK1 activity detected by protein kinase assay has prevented us from determining whether ERK5 acts upstream of PDK1 or between PDK1 and PKB. Alternatively, decreased PKB activity associated with the loss of the ERK5 signaling pathway may be caused by increased protein phosphatase 2A (PP2A) activity. PP2A has been implicated in inhibiting PKB by promoting its dephosphorylation in response to sorbitol. ${ }^{26}$

Overall, absence of ERK5 or MEK5 expression changes the kinetics of cell death in response to sorbitol. The erk5-/- and mek5-/- MEFs die quicker than their wild-type counterparts suggesting that the ERK5 cascade delays rather than inhibits the apoptotic process. JNK is most likely responsible for triggering cell death by promoting cytochrome $c$ release. ${ }^{28}$ In addition to stimulating the intrinsic mitochondrial apoptotic cascade, JNK has been shown to increase FasL expression by a mechanism that implicates the phosphorylation of 14-3-3 and the subsequent nuclear translocation of Foxo3a. ${ }^{29,30,31}$ 


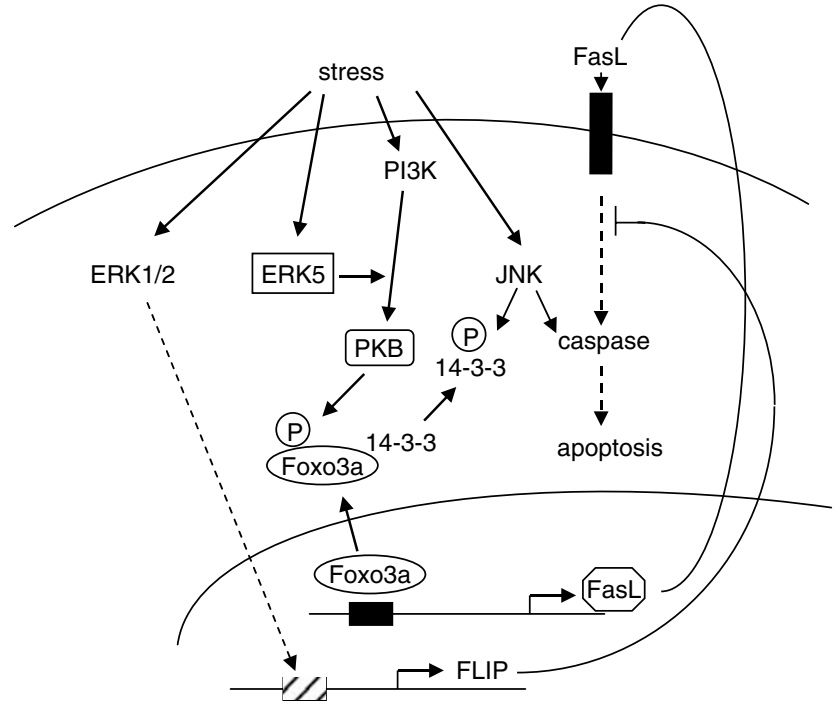

Figure 6 Regulation of cell survival by the ERK5 cascade. ERK5 contributes to inducing the phosphorylation of Foxo3a by PKB via a mechanism downstream of PI3K. Phospho-Foxo3a is sequestered in the cytoplasm via its association with 14-3-3. Under osmotic stress conditions, the nonphosphorylated form of Fox03a translocates to the nucleus where it increases fasL gene transcription. The nuclear translocation of Foxo3a is also triggered by JNK-dependent phosphorylation of 14-3-3. FasL-mediated caspase activation constitutes a positive feedback loop that enhances osmotic stress induced-intrinsic mitochondrial apoptotic cascade via JNK. ERK1/2 suppress sensitivity towards FasL-mediated apoptosis by increasing FLIP expression. The exact mechanism by which ERK5 regulates PKB remains to be identified

The antagonistic effects of JNK and ERK5 on Foxo3amediated transcription exemplify their opposite roles in the regulation of cell death and cell survival (Figure 6). Based on these studies, we propose that the survival function of the ERK5/MEK5 cascade may have a more prominent protecting effect on stresses that trigger cell death via a JNK-independent mechanism. In this situation, the level of JNK activity will be low and the ERK5 signaling pathway will be dominant over JNK.

Unlike JNK and similar to ERK5, ERK1/2 promote cell survival. ${ }^{32}$ Its ability to increase the expression of the FLICElike inhibitory protein FLIP suppresses sensitivity towards FasL-mediated apoptosis by preventing caspase 8 activation independently of PKB signaling. ${ }^{33}$ The relative importance of ERK1/2 and ERK5 in mediating cell survival is likely to be stimulus- and cell type-specific. For example, whereas ERK1/ 2 and ERK5 are both required for mediating neuronal survival in response to $\mathrm{NGF}{ }^{34}$ ERK5 is critical for the survival of endothelial cells during development. ${ }^{8,9}$ Future in vivo studies based on mutant mice in which the erk5 gene can be conditionally deleted will be crucial to establish the physiological importance of ERK5 in regulating trancriptional survival mechanisms via Foxo3a.

\section{Materials and Methods}

\section{Tissue culture and preparation of lysates}

MEFs obtained from wild-type, erk5+/loxP, erk5loxP/loxP, and mek5-/- embryos were cultured in DMEM supplemented with $10 \%$ fetal bovine serum (FBS, Invitrogen), as previously described. ${ }^{4}$ The cells were immortalized by successive passages at confluence. Transfection assays were performed using the calcium phosphate method. ${ }^{35}$ Sorbitol at $500 \mathrm{mM}$ was used to maximally activate ERK $5^{4}$ and to promote death of fibroblasts (Figure 1c and Supplementary Figure S). Time points within 1-2 $\mathrm{h}$ stimulation were chosen to study the mechanism upstream of caspase 3 , which is maximally activated by sorbitol after $3 \mathrm{~h}$ (Figure 1c). Where indicated, the cells were pretreated for $30 \mathrm{~min}$ to $1 \mathrm{~h}$ with inhibitors as follows: wortmannin (50 nM, Calbiochem), LY294002 (50 $\mu \mathrm{M}$; Calbiochem). Agonist and inhibitors were added directly to the cell culture medium

Proteins were extracted from cells in triton lysis buffer (TLB: $20 \mathrm{mM}$ Tris pH 7.4, $137 \mathrm{mM} \mathrm{NaCl}, 2 \mathrm{mM}$ EDTA, 1\% Triton-X 100, $25 \mathrm{mM}$ $\beta$ glycerophosphate, $10 \%$ glycerol, $1 \mathrm{mM}$ orthovanadate, $1 \mathrm{mM}$ phenylsulphonyl fluoride (PMSF), $10 \mu \mathrm{g} / \mathrm{ml}$ leupeptin, $10 \mu \mathrm{g} / \mathrm{ml}$ aprotinin). Extracts were clarified by centrifugation $\left(14000 \times g\right.$ for 10 min at $\left.4^{\circ} \mathrm{C}\right)$. The concentration of soluble proteins in the supernatants was quantified by the Bradford method (Bio-Rad).

\section{Viral Infections}

The adenoviruses were amplified in 293 cells, the viral solution was purified on $\mathrm{CsCl}_{2}$ gradients, and viral infectivity was determined on NIH3T3 cells. Fibroblasts were infected with recombinant adenovirus at $100 \mathrm{MOI}$, unless indicated otherwise, for $2 \mathrm{~h}$ in serum-free media, and thereafter an equal volume of DMEM containing $4 \%$ FBS was added. At $12 \mathrm{~h}$ after infection, the virus was removed, and the cells were cultured in media containing $2 \%$ FBS for a further $12 \mathrm{~h}$ unless indicated otherwise. Mockinfected MEFs used as control were placed for up to $60 \mathrm{~h}$ in $2 \%$ FBS without displaying any sign of apoptosis.

\section{Immunoblot Analysis}

Extracts $(50 \mu \mathrm{g})$ were resolved by sodium dodecyl sulfate-polyacrylamide gel electrophoresis (SDS-PAGE, 12,10 , or $8 \%$ polyacrylamide gel) and electrophoretically transferred to an Immobilon-P membrane (Millipore, Inc.). The membranes were incubated with $3 \%$ nonfat dry milk at $4^{\circ} \mathrm{C}$ for $30 \mathrm{~min}$ and then probed overnight with antibodies to ERK5 (Upstate Biotechnology), tubulin (Sigma), Cre (Chemicon), caspase 3 (Cell Signaling), JNK (Santa Cruz), phospho-JNK (New England Biolabs), Bid (R\&D Systems), Bim (Calbiochem), phospho-Foxo3a (Upstate Biotechnology), PKB (Cell Signaling), phospho-PKB (Cell Signaling), and $\mathrm{Ha}$ (Covance). Immunecomplexes were detected by enhanced chemiluminescence with anti-mouse or anti-rabbit immunoglobulin G coupled to horseradish peroxidase as the secondary antibody (Amersham-Pharmacia).

\section{Kinase assays}

PKB activity was measured in cell lysates following precipitation with a polyclonal antibody to PKB (Upstate Biotechnology) or to $\mathrm{Ha}$ (Covance). The kinase reaction was performed at $30^{\circ} \mathrm{C}$ for $30 \mathrm{~min}$ in $25 \mathrm{mM}$ HEPES $\mathrm{pH} 7.4,25 \mathrm{mM} \beta$-glycerophosphate, $25 \mathrm{mM} \mathrm{MgCl}$, $2 \mathrm{mM}$ DTT, $0.1 \mathrm{mM}$ orthovanadate containing $50 \mu \mathrm{M}\left[\gamma^{32} \mathrm{P}\right] \mathrm{ATP}(10 \mathrm{Ci} / \mathrm{mmol})$, and the peptide RPRAATF (Upstate Biotechnology) at $0.5 \mathrm{mM}$. The reaction was stopped and spotted onto $2 \mathrm{~cm}^{2} \mathrm{P} 81$ filter papers. The radioactivity bound to the filters was quantitated by liquid scintillation counting. PI3K activity was measured in cell lysates following precipitation with a polyclonal antibody to PI3K (Upstate Biotechnology) as previously described. ${ }^{36}$ Briefly, the kinase reaction was performed at $30^{\circ} \mathrm{C}$ for 
$10 \mathrm{~min}$ in $50 \mathrm{mM}$ Tris pH 7.4, $80 \mathrm{mM} \mathrm{KCl}, 10 \mathrm{mM} \mathrm{MgCl}_{2}, 2 \mathrm{mM}$ EGTA) containing $3 \mu \mathrm{g}$ phosphatidylinositol (PI), $10 \mu \mathrm{g}$ phosphatidylserine, and $50 \mu \mathrm{M}\left[\gamma^{32}{ }^{32}\right]$ ATP $(10 \mathrm{Ci} / \mathrm{mmol})$. After the reaction was stopped, the lipids were extracted, dried, resuspended in chloroform, and spotted on to a silica gel-coated glass tlc plate (Merck). Plates were resolved and exposed to autoradiography film. The radioactivity was quantitated by Phosphorlmager analysis.

\section{Reporter gene expression assay}

The reporter plasmid FHRE-luc ${ }^{16}$ was transiently cotransfected with or without expression vectors encoding wild-type (WT) or triple mutant (TM) Fox03a, ${ }^{16}$ ERK5, or MEK5. A pRL-Tk plasmid encoding Renilla luciferase was employed for monitoring transfection efficiency. Aliquots of cell lysates were assayed for firefly and Renilla luciferase activities according to the manufacturer's instructions (Promega). Cell viability was quantified by luciferase activity following transfection with the PCMV luciferase plasmid. $^{37}$

\section{Caspase assay}

Cell extracts $(20 \mu \mathrm{g})$ were lysed in $10 \mathrm{mM} \mathrm{HEPES} \mathrm{pH} \mathrm{7.5,150} \mathrm{mM} \mathrm{NaCl}$, $2 \mathrm{mM}$ EDTA containing $0.5 \%$ NP40. Extracts $(20 \mu \mathrm{g})$ were incubated with $200 \mu \mathrm{M}$ DEVD-AMC caspase 3-specific fluorogenic substrate for $1 \mathrm{~h}$. Cleavage of the substrate was measured by spectrofluorometer.

\section{Flow cytometry}

MEFs were trypsinized and incubated for $1 \mathrm{~h}$ at $4^{\circ} \mathrm{C}$ in phosphate-buffered salin (PBS) containing 5\% FBS and $10 \mu \mathrm{g} / \mathrm{ml}$ primary antibody against FasL (MFL3, BD Pharmingen). After three washes in PBS, cells were incubated for $30 \mathrm{~min}$ at $4{ }^{\circ} \mathrm{C}$ in PBS containing $5 \%$ FBS and $10 \mu \mathrm{g} / \mathrm{ml}$ fluorescein isothiocyanate (FITC)-conjugated goat anti-Armenian hamster IgG (Jackson ImmunoResearch Laboratories). Following three washes in PBS, cells were analyzed by FACScan (DAKO CYAN) with Summit 3.3 software at $488 \mathrm{~nm}$ excitation and the emission was measured with a 530/ 40 bandpass filter. Cells stained with FITC-conjugated goat anti-Armenian hamster IgG only served as internal control.

\section{ChIP assay}

Samples were prepared using ChIP-IT Enzymatic kit (Active Motif) and anti-Foxo3a antibody (Upstate Biotechnology). DNA samples recovered from DNA-Protein immunocomplexes were amplified by PCR using the following primer sets: ${ }^{38}$ promoter region containing FasL-binding site (-283/-63) 5'-GATTCAACTCCCTATGCTCAGATGTGGAG-3' and 5'-TC CAATTGGCGTCTCTGTGCTAACTGAGAAG-3'; Intron2 region, served as negative control $(+2439 /+2720) 5^{\prime}$-CAAAGGACCAGAGCCCAGGATAC AC-3' and 5'-GTTGCGATTTTGATACTTTTGTGATGG-3'.

\section{Real-time PCR}

Total RNA was isolated using the Trizol ${ }^{\mathrm{TM}}$ reagent and CDNA synthesis was carried out as previously described. ${ }^{7}$ Real-time quantitative PCRs were performed using the SYBR Green I Core Kit (Eurogentec). Primers used were: forward primer, $5^{\prime}$-AGCCCCTAAACCACAAGGTC- $3^{\prime}$ and reverse primer, 5'-TGAATACTGCCCCCAGGTAG-3' for fasL; forward primer, $5^{\prime}$-CTTGTCAAATTCTGTCAGCAACA-3' and reverse primer $5^{\prime}$-AGGTTT GCACTAGCTGAATACA-3' for foxo3a; and forward primer, $5^{\prime}$-CCAACTT GATGTATGAAGGCTTTG- $3^{\prime}$ and reverse primer $5^{\prime}$-AATTGGTCTCAAGT
CAGTGTACAGGC-3' for $\beta$-actin to generate amplicons of 135,150 , and $91 \mathrm{bp}$, respectively. PCR products were detected in the ABI-PRISM 7700 sequence detection systems (Applied Biosystems). Results were analyzed using the $2^{-\Delta \Delta G}$ methods. $^{39}$ The level of expression of mRNA was normalized to $\beta$-actin mRNA.

\section{Acknowledgements}

We are indebted to A Nagy for kindly providing us with the R1 ES cells and B Carter, C Streuli, and A Tolkovsky for their generosity in providing recombinant adenoviruses. We thank $D$ Alessi and PJ Coffer for providing us with the PKB and FHRE-luc constructs and A Whitmarsh for critically reviewing the manuscript. This work was supported by the BBSRC, and a Lister Institute of Preventive Medicine Research Fellowship to CT.

\section{References}

1. Johnson GL and Lapadat R (2002) Mitogen-activated protein kinase pathways mediated by ERK, JNK, and p38 protein kinases. Science 298: 1911-1912.

2. Lee JD, Ulevitch RJ and Han J (1995) Primary structure of BMK1: a new mammalian map kinase. Biochem. Biophys. Res. Commun. 213: 715-724.

3. Zhou G, Bao ZQ and Dixon JE (1995) Components of a new human protein kinase signal transduction pathway. J. Biol. Chem. 270: 12665-12669.

4. Wang X, Merritt AJ, Seyfried J, Guo C, Papadakis ES, Finegan KG, Kayahara M, Dixon J, Boot-Handford RP, Cartwright EJ, Mayer U and Tournier C (2005) Targeted deletion of mek5 causes early embryonic death and defects in the ERK5/MEF2 cell survival pathway. Mol. Cell. Biol. 25: 336-345.

5. Kato Y, Kravchenko V, Tapping RI, Han J, Ulevitch RJ and Lee J-D (1997) BMK1/ERK5 regulates serum-induced early gene expression through transcription factor MEF2C. EMBO J. 16: 7054-7066.

6. Kato Y, Zhao M, Morikawa A, Sugiyama T, Chakravortty D, Koide N, Yoshida T, Tapping RI, Yang Y, Yokochi T and Lee J-D (2000) Big mitogen-activated kinase regulates multiple members of the MEF2 protein family. J. Biol. Chem. 275: $18534-18540$.

7. Kayahara $M$, Wang $X$ and Tournier $C$ (2005) Selective regulation of $c$-jun gene expression by the mitogen-activated protein kinases via the TPA-responsive element and the myocyte enhancer factor 2 binding sites. Mol. Cell. Biol. 25: 3784-3792.

8. Hayashi M and Lee J-D (2004) Role of the BMK1/ERK5 signaling pathway: lessons from knockout mice. J. Mol. Med. 82: 800-808.

9. Hayashi M, Kim SW, Imanaka-Yoshida K, Yoshida T, Abel ED, Eliceiri B, Yang Y, Ulevitch RJ and Lee J-D (2004) Targeted deletion of BMK1/ERK5 in adult mice perturbs vascular integrity and leads to endothelial failure. J. Clin. Invest. 113: 1138-1148.

10. Pi X, Yan C and Berk BC (2004) Big mitogen-activated protein kinase (BMK1)/ ERK5 protects endothelial cells from apoptosis. Cir. Res. 94: 362-369.

11. Cavanaugh JE (2004) Role of extracellular signal regulated kinase 5 in neuronal survival. Eur. J. Biochem. 271: 2056-2059.

12. Alessi DR, Andjelkovic M, Caudwell B, Cron P, Morrice N, Cohen $P$ and Hemmings BA (1996) Mechanism of activation of protein kinase $B$ by insulin and IGF-1. EMBO J. 15: 6541-6551.

13. Alessi DR, James SR, Downes CP, Holmes AB, Gaffney PR, Reese CB and Cohen $P$ (1997) Characterization of a 3-phosphoinositide-dependent protein kinase which phosphorylates and activates protein kinase Balpha. Curr. Biol. 7: 261-269.

14. Sarbassov DD, Guertin DA, Ali SM and Sabatini DM (2005) Phosphorylation and regulation of Akt/PKB by the rictor-mTOR complex. Science 307: 1098-1101.

15. Datta SR, Dudek H, Tao X, Masters S, Fu H, Gotoh Y and Greenberg ME (1997) Akt phosphorylation of BAD couples survival signals to the cell-intrinsic death machinery. Cell 91: 231-241.

16. Brunet A, Bonni A, Zigmond MJ, Lin MZ, Juo P, Hu LS, Anderson MJ, Arden KC, Blenis J and Greenberg ME (1999) Akt promotes cell survival 
by phosphorylating and inhibiting a Forkhead transcription factor. Cell 96: 857-868.

17. Puthalakath H, Huang DC, O'Reilly LA, King SM and Strasser A (1999) The proapoptotic activity of the Bcl-2 family member Bim is regulated by interaction with the dynein motor complex. Mol. Cell 3: 287-296.

18. Faris M, Latinis KM, Kempiak SJ, Koretzky GA and Nel A (1998) Stressinduced Fas ligand expression in T cells is mediated through a MEK kinase 1-regulated response element in the Fas ligand promoter. Mol. Cell. Biol. 18 5414-5424.

19. Kolbus A, Herr I, Schreiber M, Debatin KM, Wagner EF and Angel P (2000) cJun-dependent $\mathrm{CD} 95-\mathrm{L}$ expression is a rate-limiting step in the induction of apoptosis by alkylating agents. Mol. Cell. Biol. 20: $575-582$.

20. Sohn SJ, Sarvis BK, Cado D and Winoto A (2002) ERK5 MAPK regulates embryonic angiogenesis and acts as a hypoxia-sensitive repressor of vascular endothelial growth factor expression. J. Biol. Chem. 277: 43344-43351.

21. Buschbeck M and Ulrich A (2005) The unique C-terminal tail of the mitogenactivated protein kinase ERK5 regulates its activation and nuclear shuttling. J. Biol. Chem. 280: 2659-2667.

22. Suhara T, Kim HS, Kirshenbaum LA and Walsh K (2002) Suppression of Akt signaling induces Fas ligand expression: involvement of caspase and Jun kinase activation in Akt-mediated Fas ligand regulation. Mol. Cell. Biol. 22: 680-691.

23. Dijkers PF, Medema RH, Lammers JW, Koenderman L and Coffer PJ (2000) Expression of the pro-apoptotic $\mathrm{Bcl}-2$ family member Bim is regulated by the forkhead transcription factor FKHR-L1. Curr. Biol. 10: 1201-1204.

24. Tang D, Okada H, Ruland J, Liu L, Stambolic V, Mak TW and Ingram AJ (2001) Akt is activated in response to an apoptotic signal. J. Biol. Chem. 276: 30461-30466.

25. Konishi $\mathrm{H}$, Matsuzaki $\mathrm{H}$, Tanaka M, Ono $\mathrm{Y}$, Tokunaga $\mathrm{C}$, Kuroda $\mathrm{S}$ and Kikkawa U (1996) Activation of RAC-protein kinase by heat shock and hyperosmolarity stress through a pathway independent of phosphatidylinositol 3-kinase. Proc. Natl. Acad. Sci. USA 93: 7639-7643.

26. Meier R, Thelen $M$ and Hemmings BA (1998) Inactivation and dephosphorylation of protein kinase Balpha (PKBalpha) promoted by hyperosmotic stress. EMBO J. 17: 7294-7303.

27. Shaw M, Cohen $P$ and Alessi DR (1998) The activation of protein kinase $B$ by $\mathrm{H}_{2} \mathrm{O}_{2}$ or heat shock is mediated by phosphoinositide 3-kinase and not by mitogen-activated protein kinase-activated protein kinase-2. Biochem. J. 336: 241-246.
28. Tournier $C$, Hess $P$, Yang DD, Xu J, Turner TK, Nimnual A, Bar-Sagi D, Jones SN, Flavell RA and Davis RJ (2000) Requirement of JNK for stress-induced activation of the cytochrome $c$ - mediated death pathway. Science 288: 870-874.

29. Sunayama J, Tsuruta F, Masuyama N and Gotoh Y (2005) JNK antagonizes Akt-mediated survival signals by phosphorylating 14-3-3. J. Cell Biol. 170: 295-304.

30. Faris M, Kokot N, Latinis K, Kasibhatla S, Green DR, Koretzky GA and Nel A (1998) The c-Jun N-terminal kinase cascade plays a role in stress-induced apoptosis in Jurkat cells by up-regulating Fas ligand expression. J. Immunol. 160: 134-144.

31. Le-Niculescu H, Bonfoco E, Kasuya Y, Claret F-X, Green DR and Karin M (1999) Withdrawal of survival factors results in activation of the JNK pathway in neuronal cells leading to Fas ligand induction and cell death. Mol. Cell. Biol. 19: 751-763.

32. Xia Z, Dickens M, Raingeaud J, Davis RJ and Greenberg ME (1995) Opposing effects of ERK and JNK-p38 MAP kinases on apoptosis. Science 270: 1326-1331.

33. Yeh J-H, Hsu S-C, Han S-H and Lai M-Z (1998) Mitogen-activated protein kinase kinase antagonized fas-associated death domain protein-mediated apoptosis by induced FLICE-inhibitory protein expression. J. Exp. Med. 188: 1795-1802.

34. Watson FL, Heerssen HM, Bhattacharyya A, Klesse L, Lin MZ and Segal RA (2001) Neurotrophins use the Erk5 pathway to mediate a retrograde survival response. Nat. Neurosci. 4: 981-988.

35. Hawkes NA and Roberts SGE (1999) The Role of human TFIIB in transcription start site selection in vitro and in vivo. J. Biol. Chem. 274 14337-14343.

36. Serunian LA, Auger KR and Cantley LC (1991) Identification and quantification of polyphosphoinositides produced in response to platelet derived growth factor stimulation. Methods Enzymol. 198: 78-87.

37. Seyfried J, Wang X, Kharebava G and Tournier C (2005) A novel MAPK docking site in the N-terminus of MEK $5 \alpha$ organizes the components of the ERK5 signaling pathway. Mol. Cell. Biol. 25: 9820-9828.

38. Jonsson $\mathrm{H}$, Allen $\mathrm{P}$ and Peng SL (2005) Inflammatory arthritis requires Foxo3a to prevent Fas ligand-induced neutrophil apoptosis. Nat. Med. 11: 666-671.

39. Livak KL and Schmittgen TD (2001) Analysis of relative gene expression data using real-time quantitative PCR and the 2(-Delta Delta $C(T))$. Methods 25 : $402-408$.

Supplementary Information accompanies the paper on Cell Death and Differentiation website (http://www.nature.com/cdd) 